\title{
Carencias Sociales: una visión desde el Proyecto de Presupuesto de Egresos de la Federación 2016
}

\author{
Social gaps: a view from the draft Expenditure \\ Budget of the Federation 2016
}

Alejandra Huerta Pineda*

\section{Resumen}

Con base en una agrupación de programas sociales federales de acuerdo a su incidencia en los indicadores de la pobreza multidimensional elaborada por el Coneval, el presente análisis muestra la evolución que ha tenido el gasto federal ejercido por carencia social y se compara con la evolución que han tenido dichos indicadores desde 2008.

El presupuesto destinado a programas sociales federales asociados a las seis carencias sociales se ha incrementado en términos reales durante los últimos años, particularmente para programas que tienen incidencia en la carencia en alimentación y acceso a servicios de salud. No obstante, solo dos carencias sociales han presentado una reducción considerable, acceso a servicios de salud, y calidad y espacios de la vivienda. En dos casos, incluso han aumentado: acceso a la alimentación y servicios básicos de la vivienda.

La evolución del presupuesto federal por carencias sociales que se presenta en este análisis puede ser de utilidad para determinar la asignación de montos en el proyecto de presupuesto para 2016 a la luz de la evolución de los diferentes indicadores de las carencias sociales de la pobreza multidimensional.

\footnotetext{
Palabras clave:

- Estructuras del Sector Público

- Gasto público

- Presupuesto
}

\begin{abstract}
Based on a group of federal social programs according to their impact on the indicators of multidimensional poverty developed by the Coneval, this analysis shows the evolution that has taken federal spending exercised by social deprivation and compared with the evolution that have had these indicators since 2008 .

The federal budget for social programs associated with the six social deprivation has increased in real terms in recent years, particularly for programs that impact on food and lack access to health services. However, only two social gaps have presented a considerable reduction, access to health services, and quality and living spaces. In two cases, they have even increased: access to food and basic housing.

The evolution of the federal budget for social needs presented in this analysis may be useful for determining the allocation of amounts in the draft budget for 2016 in the light of the evolution of the different indicators of social deprivation of multidimensional poverty.
\end{abstract}

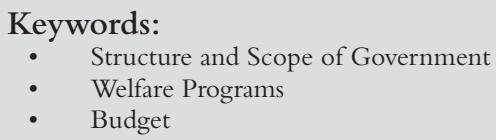

JEL: H11, M53, M61

En el marco de la discusión y aprobación del Paquete Económico 2016 se presenta a continuación un análisis del presupuesto que se ha ejercido en los programas federales que atienden en alguna medida las carencias sociales, las cuales forman parte de la medición de la pobreza multidimensional en México, que elabora el Consejo Nacional de Evaluación de la Política de Desarrollo Social (Coneval). 
La pobreza multidimensional es un concepto que engloba conceptos de bienestar económico y de derechos sociales, entendidos como carencias sociales. Una persona se encuentra en este tipo de pobreza si sus ingresos son insuficientes para satisfacer sus necesidades básicas y además tiene alguna de las siguientes carencias sociales: alimentación, rezago educativo, acceso a servicios de salud, acceso a la seguridad social, calidad y espacios de la vivienda y/o de servicios básicos de la vivienda.

Una persona tiene carencia por acceso a la alimentación si los hogares presentan un grado de inseguridad alimentaria moderado o severo, situación que implica que la alimentación no es variada y suficiente y/o no consiste de tres comidas al día.

El rezago educativo existe en alguno de los siguientes casos: 1) una persona entre 3 y 15 años no cuenta con la educación básica obligatoria y no asiste a un centro de educación formal; 2) nació antes de $1982^{1}$ y no cuenta con primaria completa; 3) nació después de 1982, tiene más de 16 años y no cuenta con secundaria completa.

Una persona tiene carencia por acceso a servicios de salud si no cuenta con adscripción o derecho a recibir servicios médicos de alguna institución, Instituto Mexicano del Seguro Social (IMSS), Instituto de Seguridad y Servicios Sociales de los Trabajadores del Estado (ISSSTE) federal o estatal, Petróleos Mexicanos (Pemex), Ejército o Marina, cuenta con Seguro Popular o servicio médico privado.

Se considera que una persona tiene acceso a seguridad social si cumple con alguna de las siguientes condiciones: 1) tiene un trabajo con acceso a las prestaciones establecidas en el artículo $2^{\circ}$ de la Ley del Seguro Social; 2) dispone de servicios médicos como prestación laboral o por contratación voluntaria al régimen obligatorio del IMSs, además del Sistema de Ahorro para el Retiro (SAR) y una Administradora de Fondos para el Retiro (Afore); 3) Para la población en general que cuente con alguna pensión o jubilación, o sea familiar de una persona dentro del hogar con acceso a seguridad social o; 4) en caso de ser adulto mayor, si es beneficiario de algún programa social de pensiones.

Se encuentran en carencia por calidad y espacios de la vivienda aquellas personas que habitan una vivienda cuyos pisos son de tierra; o con techos de lámina de cartón o desechos; o muros de lámina, material de desecho u otros similares; o tienen un nivel de hacinamiento de 2.5 personas o más por cuarto.

\footnotetext{
${ }^{1}$ Hasta 1982 la educación obligatoria era el nivel primaria.
} 
Por último, se encuentran en carencia por acceso a servicios básicos de la vivienda aquellas personas que habitan una vivienda que no tienen acceso a agua entubada directa a su vivienda; o no cuentan con drenaje; o no disponen de energía eléctrica; o su combustible para cocinar es la leña o carbón y no cuentan con chimenea.

De acuerdo con la última medición de la pobreza multidimensional de 2014 el número de personas en pobreza multidimensional aumentó en cerca de dos millones de personas de 2012 a 2014, sin embargo, también durante dicho periodo 4 de las 6 carencias disminuyeron: 3.5 millones de personas más tienen acceso a salud, 1.7 millones de personas más tienen acceso a algún tipo de beneficio de seguridad social, 1.13 millones de personas más superaron la carencia por calidad y espacios de la vivienda y 200 mil personas superaron la condición de rezago educativo. Por otro lado, la carencia por acceso a los servicios básicos de la vivienda se incrementó en 552 mil personas, y la carencia por acceso a la alimentación aumentó en 639 mil personas.

Visto desde un horizonte de tiempo más amplio, durante el periodo de 2008 a 2014 se observa que las carencias de acceso a servicios de salud y calidad y espacios de la vivienda han mostrado las disminuciones más importantes (Ver gráfica 1):

- El número de personas que no tienen acceso a servicios de salud cayó $51.4 \%$ durante dicho periodo, al pasar de 44.8 millones de personas en 2008 a 21.8 millones en 2014.

- El número de personas que no tienen acceso a espacios mínimos y calidad en la vivienda cayó $23.9 \%$, pasó de 19.4 millones a 14.8 millones durante dicho periodo. 


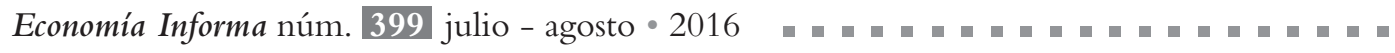

\section{Gráfica I}

\section{Carencias sociales de la pobreza multidimensional}

(millones de personas)

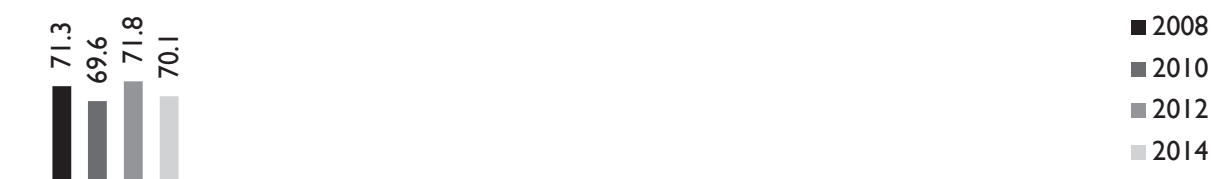

Dos carencias sociales han mostrado disminuciones moderadas: rezago educativo y acceso a seguridad social:

- El rezago educativo disminuyó 7\% al pasar de 24.1 millones de personas a 22.4 millones.

- El acceso a seguridad social se redujo en $1.16 \%$, ya que pasó de $71.3 \mathrm{mi-}$ llones de personas a 70.1 millones.

Dos carencias aumentaron durante el mismo periodo: acceso a servicios básicos de la vivienda y acceso a la alimentación.

- La población que no tiene acceso a servicios básicos de la vivienda aumentó $20.7 \%$, ya que pasó de 21.1 millones de personas en 2008 a 25.4 en 2014.

- Por último, la población que no tuvo acceso a alimentación aumentó 17.4\%, al pasar de 23.8 millones de personas a 28 millones en 2014. 
La evolución de las carencias sociales puede ser un elemento útil en la conformación del presupuesto si se tienen identificados aquellos programas que tienen incidencia en el abatimiento de las diferentes dimensiones de la pobreza, de forma que se puedan asignar montos de presupuesto acordes con las prioridades de política pública y los mayores rezagos.

En el contexto del Presupuesto Base Cero, el Coneval elaboró el documento, "Consideraciones sobre el Proceso Presupuestario 2016"2, en el cual hace un análisis de la incidencia que tienen los distintos programas en la atención de los derechos sociales establecidos en la Ley General de Desarrollo Social y que son utilizados en la medición de pobreza, asignándoles una prioridad alta, media o baja en función del impacto que pudieran tener sobre el ingreso y la reducción de las carencias sociales. ${ }^{3}$

En términos generales, el criterio para asignar el nivel alto de prioridad se basó en que el programa repercuta directamente en los indicadores de la carencia, medido a través de la Encuesta Nacional de Gasto de los Hogares (ENIGH) o a través de evidencia en las evaluaciones del programa. Los programas con prioridad media fueron aquellos cuyos bienes y servicios otorgados repercuten sobre la carencia, sin embargo no se cuenta con mediciones o no existen evaluaciones que generen evidencia del impacto. En algunos casos se consideran los programas que inciden sobre el ingreso, ya que se asume que de manera indirecta influye sobre la eliminación de la carencia. Por último, a los programas que se les asigna una prioridad baja son aquellos con un efecto posible o potencial sobre la carencia, pero que no cuentan con medición o alguna evaluación que permita contar con evidencia de dicho efecto y/o en aquellos casos en que la población beneficiada no necesariamente es la que vive en pobreza. ${ }^{4}$

De esta manera, el Coneval identifica 30 programas con base en el PEF 2015 que contribuyen en mayor o menor medida a atender alguna de las carencias sociales. Con base en dicha clasificación y considerando las modificaciones que surgieron de la Estructura Programática ${ }^{5}$ se agrupó el presupuesto destinado a programas que atienden cada una de las carencias sociales que se

\footnotetext{
${ }^{2}$ Disponible en: http://www.coneval.gob.mx/Evaluacion/IEPSM/Documents/Consideraciones_presupuestales_2016.pdf

${ }^{3}$ En esta nota se analizan los programas que de acuerdo al Coneval tienen incidencia en las carencias sociales.

${ }^{4}$ El criterio de clasificación varía ligeramente según la carencia, para mayor detalle se recomienda consultar el documento citado.

${ }^{5}$ Para mayor detalle de la nueva estructura programática, se recomienda consultar: http://www. senado.gob.mx/ibd/content/productos/docs/estructura_programatica2016.pdf
} 
Economía Informa núm. 399 julio - agosto • 2016 - "

ejerció en 2010, 2012, 2014 y el del Proyecto de Presupuesto de Egresos de la Federación para 2016 (Ppef2016) (Ver gráfica 2).

Durante el periodo de 2010 a 2014 el presupuesto para programas que abaten de manera directa la carencia de rezago en seguridad social presentó un incremento de $234.5 \%$ real. En este presupuesto se consideran solo dos programas: Pensión para Adultos Mayores y el Seguro de Vida de Jefas para Familias, que surgió en 2013. El primer programa tiene una contribución directa en abatir la carencia, ya que dicho indicador considera el acceso a programas sociales de pensiones; mientras que el segundo tiene una contribución indirecta ya que los seguros de vida se contemplan como beneficios de la seguridad social, pero es necesario que esta población se encuentre ocupada, jubilada o pensionada. A pesar del aumento en este presupuesto, la caída en dicha carencia fue de solo $1.6 \%$ de 2008 a 2014, lo que se explica en parte porque el Programa de Adultos Mayores solo abate la carencia en un grupo de la población, personas mayores de 60 años.

\section{Gráfica 2}

Presupuesto de programas con incidencia en las carencias sociales (MMDP de 2016)

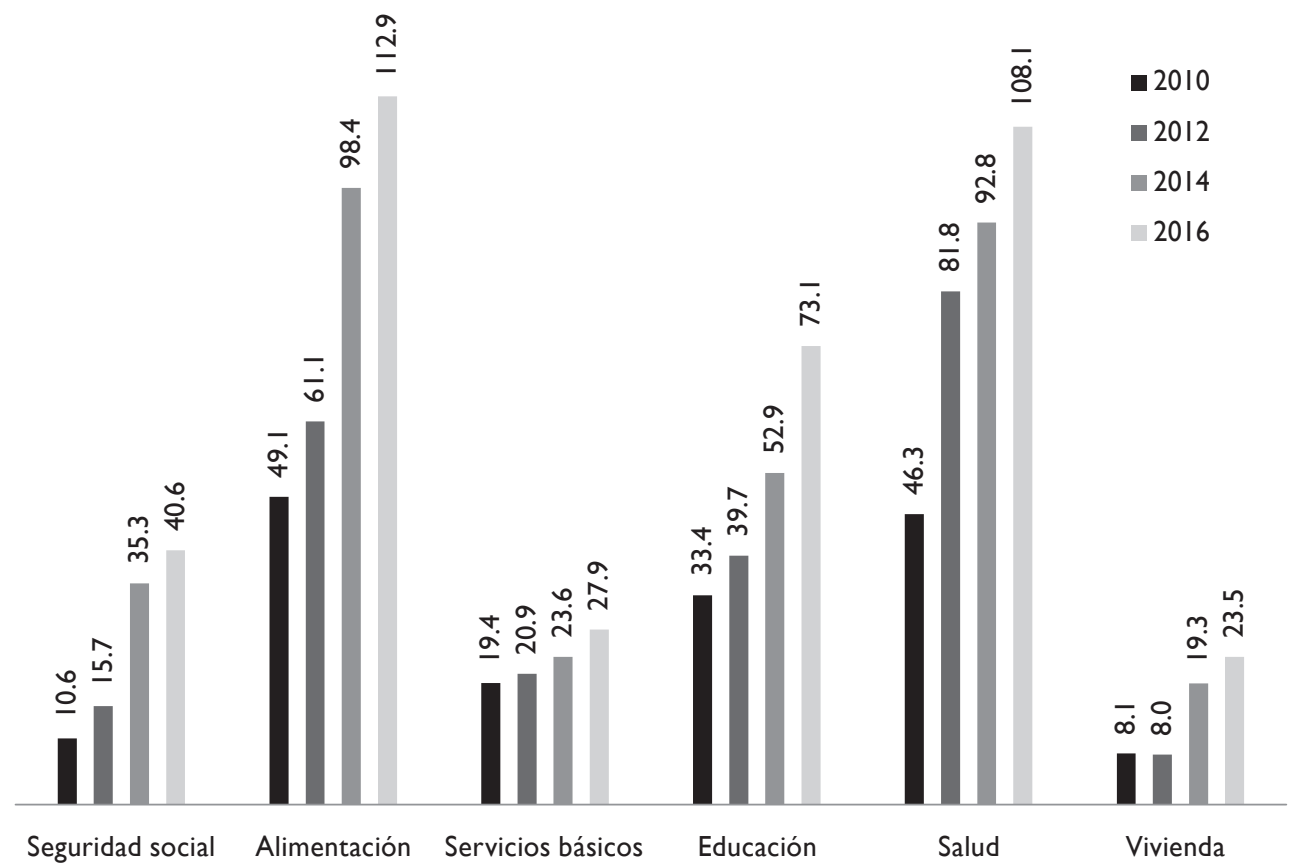

Fuente: Elaboración del IBD con base en los datos de la Cuenta Pública 2010, 2012, 2014 y PPEF 2016. Nota: El presupuesto entre carencias no es excluyente, ya que algunos programas atienden componentes de varias carencias y por tanto pueden estar contabilizados en más de una de ellas. 
En el PPeF2016 se propone un aumento de 6.28\% real para el Programa de Seguro de Vida de Jefas de Familia y una disminución de 4.25\% real en el Programa de Pensión para Adultos Mayores para 2016 (Ver Tabla 1).

El presupuesto de programas que atienden la carencia en alimentación aumentó $100.5 \%$ real de 2010 a 2014, mientras que, la carencia por acceso a la alimentación aumentó $17.4 \%$ de 2008 a 2014. En este grupo de consideran 20 programas, la mayoría de la Secretaría de Desarrollo Social (Sedesol), que contribuyen al abatimiento de dicho indicador ya sea mediante la entrega directa de alimentos como es el caso de Diconsa y Liconsa o mediante la entrega de subsidios monetarios mediante los cuales la población beneficiaria puede tener acceso a más y mejores alimentos (ver Tabla 1). Es importante mencionar que la mayoría de estos programas no tienen el único objetivo de mejorar la alimentación de sus beneficiarios, sin embargo, se espera que esta población utilice los apoyos para atender sus necesidades básicas, entre ellas la alimentación. En el PPEF2016 se propone aumentar el presupuesto de todos estos programas, excepto del Programa de Adultos Mayores, por lo que en el agregado el monto propuesto para programas que contribuyen al abatimiento de la carencia implica un incremento de $1.73 \%$ en términos reales.

Los programas que contribuyen a abatir la carencia de servicios básicos de la vivienda tuvieron un incremento real de su presupuesto agregado de $21.1 \%$ de 2010 a 2014, mientras que la carencia respectiva se incrementó $20.7 \%$ de 2008 a 2014. En esta carencia se agrupan 5 programas federales, dos de ellos se integrarán en 2016 en el Programa de Infraestructura, a cargo de la Secretaría de Desarrollo Agrario, Territorial y Urbano (Sedatu), para el cual se propone una disminución de 2.3\% real respecto de lo aprobado en 2015. Dos programas de la Secretaría de Medio Ambiente y Recursos Naturales (Semarnat) con acciones de drenaje y agua potable, se agrupan en el Programa de agua potable, alcantarillado y saneamiento, para el cual se propone un incremento en su presupuesto de 6.3\%. Finalmente, el Programa de Infraestructura Indígena presenta un incremento de 6.3\% en términos reales respecto del aprobado para 2015.

El Coneval identificó 7 programas federales que contribuyen al abatimiento del rezago educativo, los cuales en su conjunto presentaron un incremento de $58.5 \%$ real de 2010 a 2014, mientras que el rezago educativo disminuyó 7\% de 2008 a 2014. Dentro de este grupo se encuentran 3 programas de la SEP, Atención a la Demanda de Educación para Adultos, Prestación de Servicios de Educación Inicial y Básica Comunitaria (Conafe) y el Programa Nacional de Becas; 3 programas de la Sedesol con acciones que contribuyen a aumentar la asistencia y permanencia escolar, el Programa de Atención a Jornaleros Agrícolas, Prospera, 
Seguro de Vida de Jefas para Familia; y el Programa de Apoyo a la Educación Indígena. Para todos estos programas se proponen presupuestos para 2016 con un incremento de entre 6.3 y $6.7 \%$ en términos reales respecto de 2015 .

El presupuesto en programas federales que se ha destinado a salud creció 100.3\% de 2010 a 2014, lo cual se encuentra en línea con la disminución de la carencia por acceso a servicios de salud que fue de $51.4 \%$ de 2008 a 2014. En este grupo se encuentran 5 programas de la Secretaria de Salud, 3 de los cuales tienen una contribución directa al abatimiento de la carencia: Seguro Popular, Seguro Médico Siglo xxi y Unidades Médicas Móviles. Para 2016 se plantea una disminución de $0.83 \%$ en términos reales para el Programa Seguro Popular, y un aumento de $2.25 \%$ real para el nuevo Programa Fortalecimiento a la atención médica que integrará para 2016 al Programa de Unidades Médicas y el Fortalecimiento de las Redes de Servicios de Salud.

En calidad y espacios de la vivienda el incremento en el presupuesto ejercido en el periodo 2010 a 2014 fue de $137.8 \%$ en términos reales, mientras que la carencia disminuyó $1.13 \%$ de 2008 a 2014. A dicho indicador contribuyen 4 programas federales, pero de acuerdo al documento de Coneval, solo el Programa para el Desarrollo de Zonas Prioritarias contribuye de manera directa. Este programa fue agrupado con el Programa Hábitat y en su conjunto presentan un propuesta de presupuesto que implica una disminución de $2.3 \%$ en términos reales para 2016. Por otro lado, los Programas de Vivienda Digna y Vivienda Rural se integrarán en el Programa de Apoyo a la Vivienda y en su conjunto tendrán un incremento de 6.29\% real. Finalmente para el Programa de Esquema de Financiamiento y Subisidio Federal para Vivienda a cargo de la Sedatu se propone un incremento de $25.8 \%$ en términos reales, el mayor incremento propuesto dentro del grupo de programas que contribuyen al abatimiento de las carencias sociales.

\section{Conclusiones y consideraciones finales}

La evolución de las carencias sociales no podría atribuirse totalmente a un aumento en el presupuesto de los programas federales, ya que existen otros programas de orden estatal o municipal, así como fondos otros ramos federales con objetivos similares. Pero principalmente, debe considerarse que la evolución de la pobreza y sus carencias se explica en gran medida por el crecimiento y desarrollo económico del país.

No obstante, por la forma como se encuentran definidas las carencias sociales, en algunos casos la contribución de los programas federales para dis- 
minuir las carencias es inmediato, tal es el caso del Seguro Popular o del Programa de Pensión de Adultos Mayores, ambos con una incidencia directa en las carencias de acceso a servicios de salud y de seguridad social. En ambos casos la propuesta de presupuesto para 2016 implica una disminución en términos reales para ambos, aun cuando existen 21.8 millones de personas en carencia por acceso a salud y 70.1 millones en carencia por acceso a seguridad social, de acuerdo a la última medición de 2014. En este contexto, es necesario garantizar que las disminuciones propuestas no afectarán las entregas de apoyos o incluso las metas sexenales establecidas en los Programas Sectoriales.

A reserva de llevar a cabo un análisis más riguroso de la incidencia del presupuesto federal en la evolución de las carencias sociales, las cifras presentadas sugieren que la caída que ha presentado la carencia por acceso a salud se atribuye en gran medida al aumento en el presupuesto destinado a este grupo de programas durante los últimos años y en forma particular al del Seguro Popular.

El Programa de Adultos Mayores también contribuye en forma directa a abatir la carencia de seguridad social, pero solo para un subgrupo de la población, los adultos mayores de 60 años, por lo que su contribución a mejorar dicho indicador es limitada. En este sentido, es necesario también reforzar otras medidas de política pública de generación de empleos con acceso a Seguridad Social que contribuirán a la disminución de dicha carencia en otros grupos de la población.

Por otro lado, la carencia alimentaria es la segunda más importante en términos de población, después de la seguridad social, ya que afecta a 28 millones de personas. El presupuesto para programas que tienen incidencia en este indicador ha aumentado en forma importante en los últimos años, en gran parte por la implementación del Programa de Comedores Comunitarios a partir de 2014. En este contexto, para 2016 se propone una vez más un incremento importante al presupuesto de este tipo de programas.

Los programas que contribuyen a disminuir el rezago educativo, mediante incentivos a la asistencia y permanencia escolar, y el abatimiento del rezago educativo de los adultos, también han incrementado su presupuesto de manera considerable, sin embargo, se observa un avance moderado en dicho indicador.

Asimismo, los programas de vivienda han presentado incrementos considerables en su presupuesto en los últimos años y es de hecho la carencia con la incidencia más baja en 2014, pues solo 14.8 millones de personas padecen dicha carencia. Sin embargo, para 2016 se propone un incremento de $25.8 \%$ 
Economía Informa núm. 399 julio - agosto • 2016

para el Programa de Esquema de Financiamiento y Subsidio Federal para la Vivienda, el más alto de todo este grupo de programas.

Finalmente, para los Programas que atienden el rezago de servicios básicos de la vivienda, una de las dos que incrementaron en 2014, se han presentado incrementos más moderados en presupuesto, 21.1\% de 2010 a 2014 y para 2016 se propone incluso una disminución al Programa de Infraestructura (antes Programa de Desarrollo de Zonas Prioritarias y Hábitat) de 2.3\%, mientras que para el resto de los programas que inciden en esta carencia se propone un incremento en línea con el resto de los programas, es decir, 6.3\% en términos reales.

En un contexto de bajo crecimiento económico y austeridad fiscal, es importante que para la toma de decisiones en la aprobación del presupuesto de egresos se considere la evolución de las carencias sociales, de forma que se prioricen aquellos programas con mayor incidencia en los indicadores, en aquellos que no han mostrado un buen desempeño durante los últimos años, o en aquellos con los niveles más altos. 
Alejandra Huerta Pineda

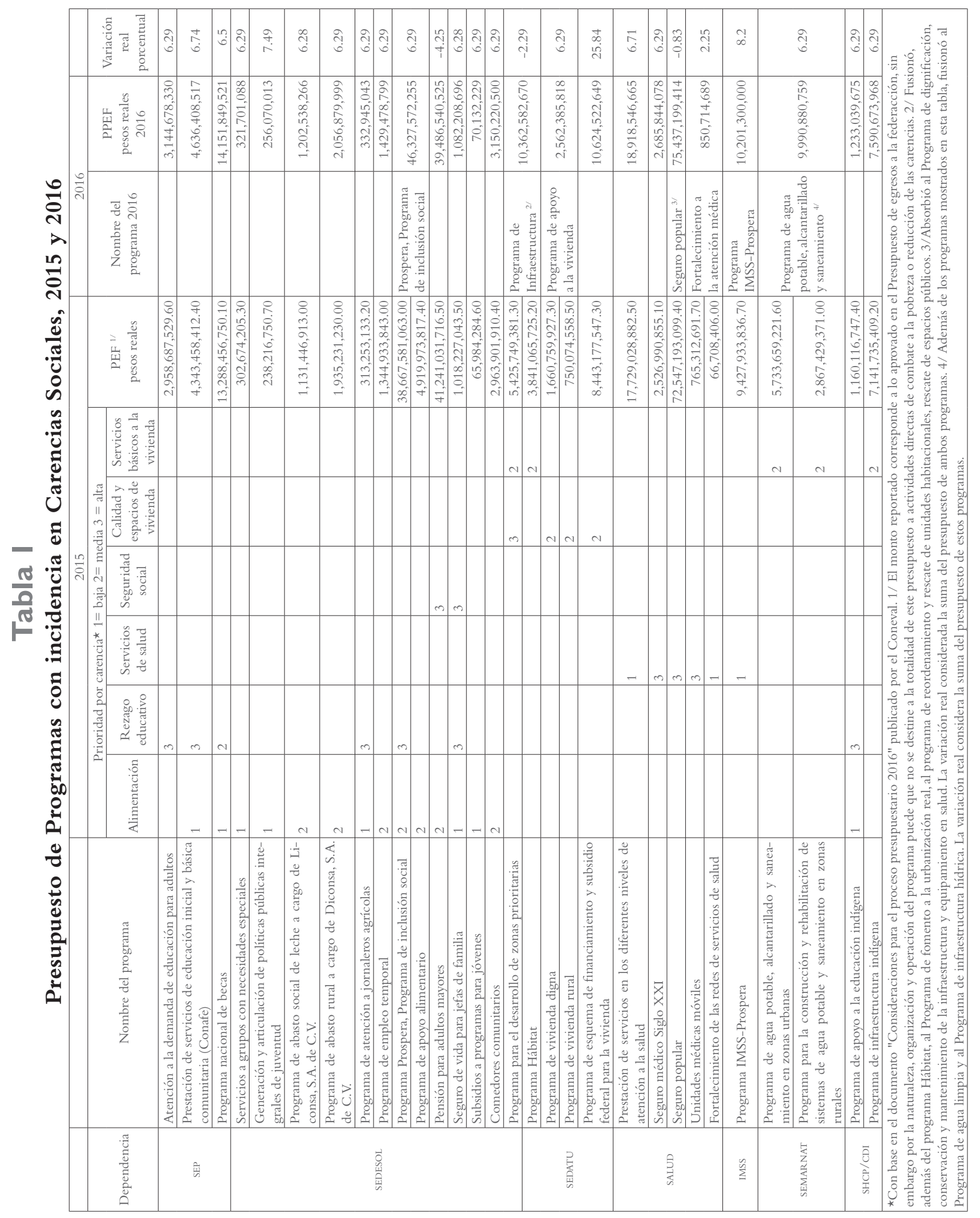

